\section{OP42 GLYCAEMIC LOAD AND INDEX IN PREGNANCY ARE ASSOCIATED WITH POSTNATAL, BUT NOT PRE- PREGNANCY, DEPRESSIVE SYMPTOMS; LONGITUDINAL DATA FROM THE SOUTHAMPTON WOMEN'S SURVEY}

\begin{abstract}
${ }^{1,2} \mathrm{H}$ Inskip*, ${ }^{3} \mathrm{H}$ Okubo, ${ }^{1} \mathrm{~S}$ Crozier, ${ }^{1,2} \mathrm{C}$ Cooper, ${ }^{1,2}{ }^{2} \mathrm{~K}$ Godfrey, ${ }^{1,2} \mathrm{~S}$ Robinson, ${ }^{1} \mathrm{~J}$ Baird. ${ }^{1} M R C$ Lifecourse Epidemiology Unit, University of Southampton, Southampton, UK; ${ }^{2}$ NIHR Southampton Biomedical Research Centre, Univ of Southampton and Univ Hospital Southampton NHS Foundation Trust, Southampton, UK; ${ }^{3}$ Department of Health Promotion, National Institute of Public Health, Saitama, Japan
\end{abstract}

\subsection{6/jech-2017-SSMAbstracts.42}

Background High glycaemic load (GL) and glycaemic index (GI) diets have been linked to poor health. In cross-sectional studies, associations between GL and GI and psychological disorders have also been identified, though the direction of association has not always been consistent. Few longitudinal data exist and none in relation to associations between GL and GI during pregnancy and postnatal depression. We aimed to assess whether higher GL and GI during pregnancy were associated with increased risk of postnatal depressive symptoms using data from the Southampton Women's Survey (SWS).

Methods The SWS is a population-based cohort study of 12583 women aged 20-34 years assessed when not pregnant; those enrolled during the latter half of the recruitment period completed the General Health Questionnaire (GHQ-12), a short screening instrument for depressive symptoms. Women who became pregnant $(n=3,158)$ were followed up. Six months post-partum, mothers completed the Edinburgh Postnatal Depression Scale questionnaire to determine postnatal depressive symptoms. Established cut-offs for each depression scale were used to determine presence of depressive symptoms before and after pregnancy. At 11 and 34 weeks' gestation, diet during the preceding 3 months was assessed using an interviewer-administered food-frequency questionnaire, from which GL and GI were determined. Prevalence ratios (PRs) for postnatal depressive symptoms were obtained using Poisson regression with robust variance. Potential confounding factors were identified through a Directed Acyclic Graph, namely age, educational attainment and smoking during pregnancy. Linear regression was used to assess the relationship between pregnancy GL and GI and pre-pregnancy depressive symptoms.

Results Postnatal depression data were available for 2856 women, with 2038 and 2429 of them having GL and GI data at 11 and 34 weeks' gestation respectively. In univariate analyses, postnatal depressive symptoms were positively associated with GL at both time points and GI at 34 weeks' gestation, but were strongest for 34 weeks' GL: PR 1.12 per $100 \mathrm{GL}$ units (95\% CI: 1.04-1.20). After adjustment for confounders, 34 weeks' GL was the only measure associated with postnatal depressive symptoms: PR 1.09 per $100 \mathrm{GL}$ units (95\% CI: 1.01-1.17). Notably, there was no association between prepregnancy depressive symptoms derived from the GHQ12 and either GL or GI.

Conclusion These findings suggest that improving diet in pregnancy, particularly lowering GL in late pregnancy, might protect against postnatal depressive symptoms. Pre-pregnancy depressive symptoms were not associated with GL and GI in pregnancy indicating that reverse causation is unlikely.

\section{OP43 NETWORK META-ANALYSIS OF EMPIRICALLY DERIVED COMPONENT CLASSES IN PARENTING INTERVENTIONS FOR CHILD CONDUCT DISORDER}

${ }^{1} \mathrm{GJ}$ Melendez-Torres*, ${ }^{2,3} \mathrm{P}$ Leijten, ${ }^{3} \mathrm{~W}$ Knerr, ${ }^{3} \mathrm{FEM}$ Gardner. 'Warwick Medical School, University of Warwick, Coventry, UK; ${ }^{2}$ Child Development and Education, University of Amsterdam, Amsterdam, NL; ${ }^{3}$ Department of Social Policy and Intervention, University of Oxford, Oxford, UK

\subsection{6/jech-2017-SSMAbstracts.43}

Background Child conduct disorders have important long-term public health sequelae, including higher risk of substance use and violence in adolescence and higher risk of mental health problems in adulthood. Parenting interventions informed by social learning theory have been consistently shown to be effective for reducing child disruptive behaviour, but the diversity and variety of interventions prevents a clear understanding of why interventions are differentially effective. Intervention components rarely exist in isolation, and interventions may be best understood in terms of the combinations of components they include rather than any one component. We tested if components of social learning theory-based parenting interventions for child disruptive behaviour outcomes formed underlying groupings across trials, and analysed how these groupings were associated with differential intervention effectiveness.

Methods We searched existing systematic reviews across a variety of platforms and updated searches in recent reviews. We included randomised controlled trials of parenting interventions for child conduct disorder measuring child disruptive behaviour outcomes. We developed and applied an exhaustive 19-component scheme against all trials. We estimated a latent class model for components in each trial arm and chose the best-fitting model. We subsequently took 20 draws from the probability distribution of the latent classes for each arm, network meta-analysed each draw, and combined findings from each draw using Rubin's rules.

Results We included 151 trials with 329 arms comparing relevant parenting programmes against each other, against treatment as usual or against no treatment. Across 19 components, our latent class analysis identified five intervention types (four active, one no treatment). As compared to no treatment, 'relationship-building' was most effective at reducing child disruptive behaviour (Cohen's d -0.71 , 95\% CI [-0.90,-0.53]) but 'parental self-management' (-0.54, [-0.76,-0.32]), 'breaking coercive cycles' $(-0.53,[-0.74,-0.33])$ and 'all components' $(-0.49,[-0.68,-0.29])$ were effective as well. There was some evidence that 'relationship-building' was more effective than other active intervention types but confidence intervals for these comparisons were broad.

Discussion We brought together two types of methods, latent class modelling and network meta-analysis, to examine how intervention classes are associated with differential intervention effectiveness. This is the first analysis of its kind. While all active intervention classes were better than no treatment and no active intervention class was better than any another, we did not find that using all possible components in the same intervention was associated with increased effectiveness as compared to more spare groupings of components. 